\title{
Extending the Use of Games in Health Care
}

\author{
Carolyn Watters, Sageev Oore $\dagger$, Michael Shepherd, Azza Abouzied, Anthony Cox, \\ Melanie Kellar, Hadi Kharrazi, Fengan Liu, and Anthony Otley \\ Faculty of Computer Science \\ Dalhousie University \\ Halifax, Nova Scotia, \\ Canada \\ $\dagger$ Department of Math and \\ Computing Science \\ Saint Mary's University \\ Halifax, Nova Scotia, Canada \\ $\$$ Pediatric Gastroenterology \\ IWK Children's Hospital \\ Halifax, Nova Scotia \\ Canada \\ Email:watters@cs.dal.ca
}

\begin{abstract}
Digital games have the ability to engage both children and adults alike. We are exploring the use of games for children with long term treatment regimes, where motivation for compliance is a key factor in the success of the treatment. In this paper, we describe the game framework we are building for this purpose. This framework is meant to support the long term use of a gaming world for children with three main goals: (a) provide easy and continual gaming access on a range of computing appliances including small screen devices; (b) offer games that can be personalized and are adaptable based on the child's interests or specific illness; and (c) maintain novelty and interest in the treatment over time. This framework not only provides a benefit to the children involved, but also provides user data to the coaches, clinicians, and health researchers involved in the child's treatment regime.
\end{abstract}

\section{Introduction}

Games have become very popular for capturing the attention of children in the promotion of healthy lifestyles to help them learn about a variety of health conditions and treatments [7; 14]. In this research we are interested in designing the next generation of health related games: games that move beyond the educational phase to the longer term support of children with chronic conditions. These children have different needs related to their individual condition and individual treatment regimes often on a daily basis over extended periods of time. The overall question we are trying to answer is "can games be used to motivate these children by reinforcing healthy choices, reminding of treatment specifics, distracting their attention, and maintaining more complete health status and treatment records?" In this paper we examine the design of game artifacts that have potential to motivate continued use in this context.

The success of digital games across a broad demographic, has led researchers to speculate that game interaction can be used to advantage in health contexts. This is based on an observation made by Turkle [15] that users of SimCity liked it because "even though it is not a video game, it plays like one." Supporting high levels of motivation in the players is crucial for young patients facing months and years of treatment. Games, whether single player or collaborative provide players with the autonomy to practice, use the computer as a coach, and yes, zone out.

While the focus of game play is largely entertainment, it is entertainment that is built on challenges, and so the adaptation of game structures for use in other contexts has appeal where values of self motivation, learning, skill practice, and meeting challenges also exist. Chapman [3] suggests that there should be increasing emphasis on the learner "situating" themselves in the world of study, to explore the possibilities in other worlds, and the view concepts and constructs from other perspectives, even take on multiple roles. Games provide players the ability to do this.

Currently, however, most health related games target the initial period after diagnosis when the child and family need to learn a great deal about the disorder, treatment, and effects of treatment. The long term treatment phase, which is much more personal, data intensive and dynamic, has to-date not really benefited from the use of games. The design of games 
for this extended period is, in some ways, more challenging than the short term engagement needed for the interactive learning of the diagnostic phase.

In the long term treatment phase, the user would be expected to return to the game frequently over longer periods of time. Over time, the child may mature and be ready for more sophisticated game interactions. Also, the particular treatment and characteristics of the disorder may change. Throughout this phase, a significant amount of data must be collected and stored on an ongoing basis. The goals of games in the treatment phase may, however, be closer to entertainment goals than the educational goals in several key ways; reinforcement of learned skills, new challenges built on changing data and achievements, monitoring of status, and commitment of the player to continue playing over multiple sessions.

This work reported in this paper is part of a three year project [13] to examine the role of games and simulations in improving learning outcomes in health related areas. Games engage users in a way that educational material does not. Therefore, we need to learn from games by building interfaces that are more transparent, more engaging, and foster community building. There is a similarity in the goals of treatment objectives and digital games, including motivation to deal with the treatment, engagement, persistence in sticking with the treatment, skill improvement, and strategies to cope with difficulties, for example. Our goal is to provide and test a game architecture that supports a new generation of health games by retaining the engagement of entertainment games and at the same time supporting long term modification of user behaviour to improve health outcomes.

\section{Why Games?}

One of the most significant factors in good health outcomes for children with long term or chronic disorders is consistent compliance with the treatment regime, even when they are not experiencing direct effects. Our working hypothesis is that the ubiquitous digital game, now considered a mass medium [17], can be exploited to achieve these health objectives. That is, the factors that make digital games so engaging can be applied usefully in the health contexts where motivation and engagement are important factors in the successful management of chronic conditions.

Games differ from most applications we use in their use of visual, textual, and auditory channels for feedback, scaffolding challenges, visible goal indicators, overviews and schematics, and ease of learning [5]. This is in stark contrast to most of the applications we use. The process of learning how to play, how to improve skills, and how to succeed is much more natural in most games than in other software applications. Games may use sample play, hints or avatars for learning by watching. Few games rely on formal instruction or courses to get the player involved. Most games support scaffolded challenges with easier levels used to develop the skills needed at more advanced levels. One of the appeals of games is the ease with which the user can personalize and customize the games in ways that are easily reversible and without risk for the user. Game interfaces have brought a level of fluidity and contextual grounding that is largely missing from most other application interfaces.

Games are designed to generate high levels of motivation and engagement in the players. Game players display intensity in the interaction, devotion (compulsion) to the game, and replay just for fun. In a previous study, Watters and Duffy [16] proposed a framework of motivational constructs, found in games, which are directly applicable to the design of interactive health software. The framework includes three dimensions of constructs; self-regulation, relatedness, and competency. Increasing selfregulation or autonomy has been shown to increase the motivation of the user to continue with the task. In games this is accomplished through easy to use navigational schema, self-pacing, attainable goals, and easy access to help when needed. Relatedness, which includes role playing, narrative themes, and personalization has been shown to increase motivation and persistence in children. Games support selfefficacy and self-confidence by providing scaffolding for tasks with meaningful successes early on, open ended challenges, and informative and timely feedback.

If these attributes of games can be kept intact throughout an adaptation of games from strictly entertainment to health related goals then an opportunity exists to engage children in the long time responsibility for their own condition. That is, we can build games that function like personalized coaches. Like games, the success of health software depends not only on the quality of the content but also on the engagement of the user and the motivation for that user to interact with the system in a meaningful way to learn new concepts, master new skills, and monitor treatment regimes that may extend over weeks or months. 


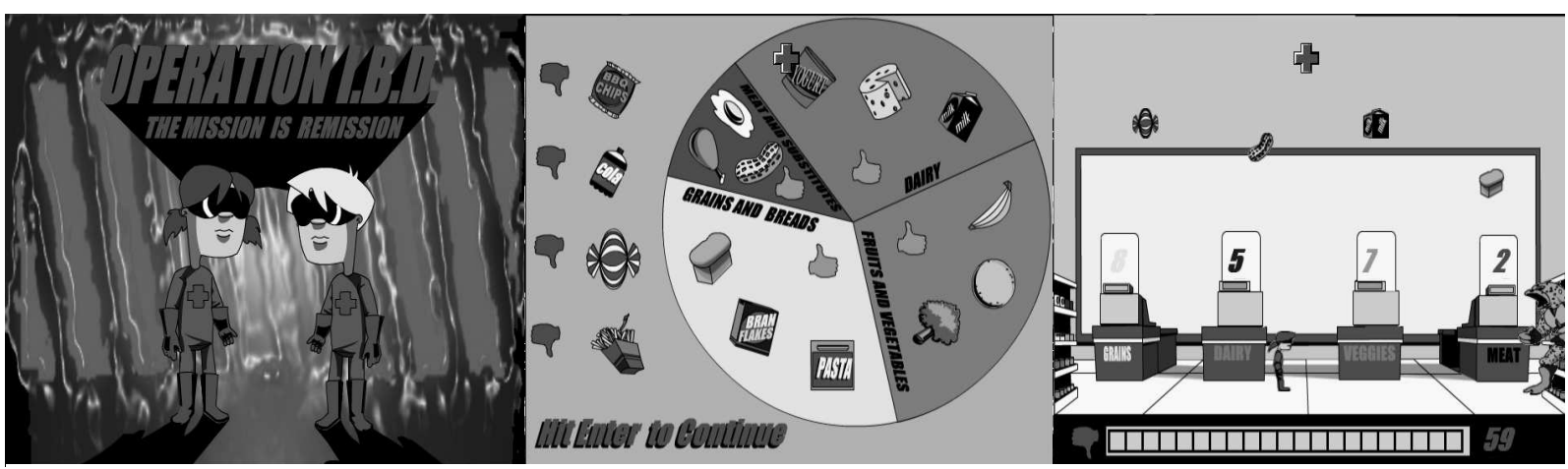

Figure 1. Choosing a balanced diet challenge in the IBD game.

\section{Games in Health}

Games are targeted for increasing the motivation of patients in three areas. First, to increase a patient's motivation to engage in learning the ins and outs of their condition and its treatment. Second, to be used as a tool in distraction therapy for pain and anxiety. Third, to encourage young patients to continue with their treatments over longer treatment regimes. The Starbright world $[10 ; 14]$ is an example of communication network used inside hospitals to exploit the use of communication and entertainment for children with serious diseases to help them cope with their treatments.

\subsection{Games for Learning}

The use of games to encourage the learning of health content has become very popular in the last five years. The idea is that game scenarios and game interactions will increase the motivation of children to engage in the educational content. Studies have shown that children who know more about their disorder have better health outcomes. For example, Bronkie the Bronchiasaurus [2] is a game that helps children learn about asthma and learn more about managing their asthma. Research showed that the children who had completed the game understood the impact of decisions they made and overall made better choices than the children who did not have access to the game. Furthermore, the children who used the game to learn about asthma treatments were found to have $40 \%$ fewer hospital emergency room.

Operation IBD (shown in Figure 1) is a game developed for 6-10 year olds to reinforce a learning component for children diagnosed with Irritable Bowel Disease (IBD). This was as part of a web site [6] of material to help children learn how to manage the symptoms of their condition. The goal of this game was to reinforce the material given in web based interactive lessons. Several lessons were learned about making games from this first work. First, making games is very expensive; second, graphics and audio are essential; and third the challenges must be real and require skill.

Although games for education are becoming more and more popular, developing games for children that appeal to both genders can pose some challenges. Cooper and Weaver [4] state that there is a striking difference in how girls and boys prefer to learn using technology. Therefore, we want to be able to provide variations of the same game that will appeal to both genders.

\subsection{Games as Distraction}

Games have also been used successfully in pain treatment as a distraction [4;9] and for pre-surgery to reduce anxiety [12]. In Patel's [12] study of 26 children, 4 to 12 years old, the use of a GameBoy outperformed the use of tranquilizers in the reduction of anxiety.

\subsection{Games as Coach}

The use of games related to the long term treatment strategy for children with chronic disorders has not been pursued to the same degree as those for the initial learning phase. There is evidence that children are quite willing to use electronic devices for health related purposes. Palermo et al [11] used PDAs for children with headaches or juvenile arthritis to keep their daily journals. They found that in a study with 60 children (ages 8 to 16) that the children with the PDA electronic diary complete the diary on more days than the children using the paper journal, 6.6 days versus 3.8 days. Furthermore, the children using the PDA made fewer errors. Glucoboy [8] is a device plugged 


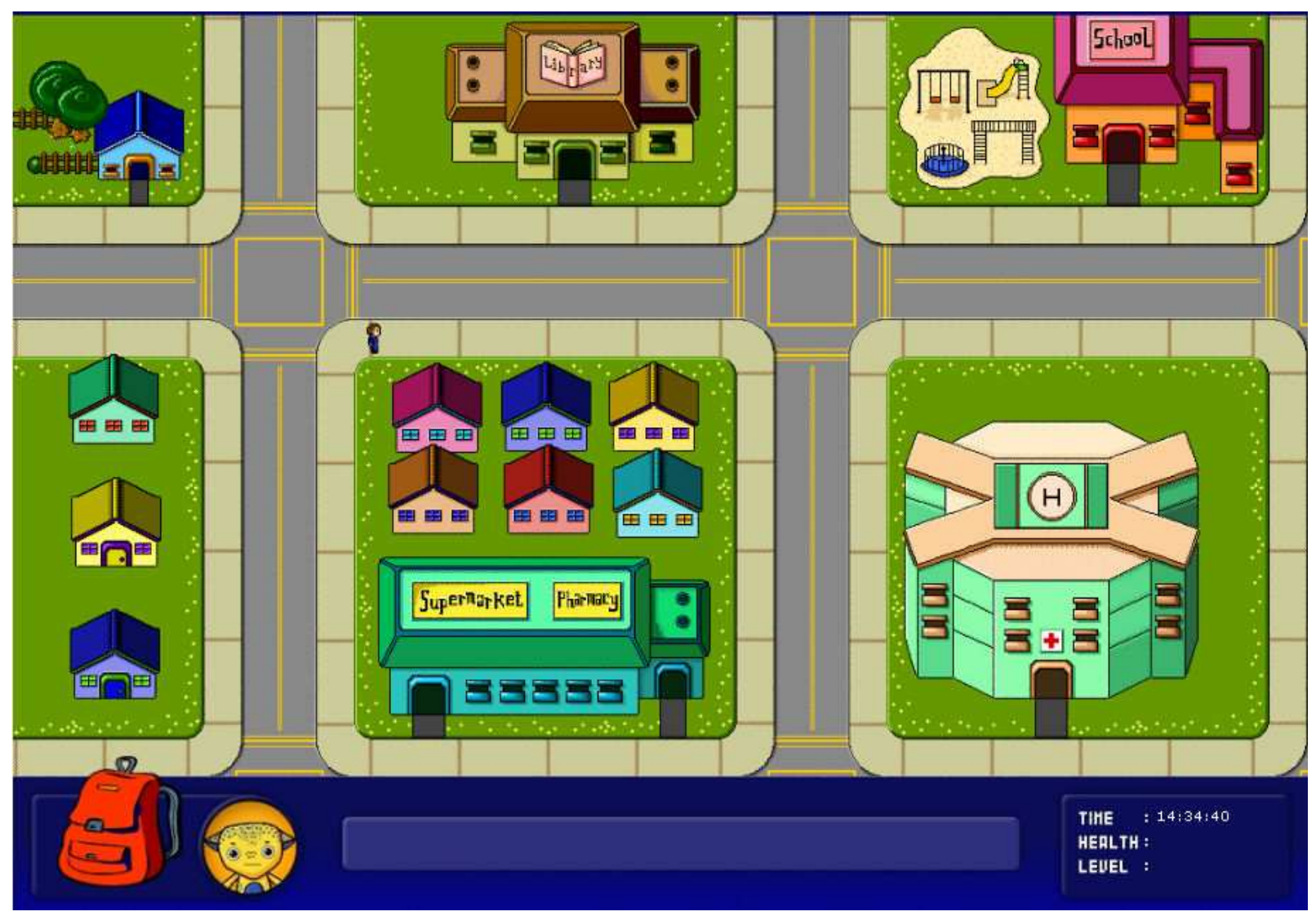

Figure 2. The city scape.

into Gameboys to help children with diabetes monitor their blood glucose levels.

Once the patients are past the initial learning phase after diagnosis, we propose the use of games to support the child in the following ways; reinforcement of information gained in initial phase, distraction as coping strategy, reminder system, encourage skill practice, and recording of treatment and status data. Long term engagement means that games will be played over and over as the treatment progresses, the child ages, and behaviour improves or regresses.

In this research, we are interested in the use of games to improve the long term behaviour of children with respect to their engagement in the adherence to their treatment regime. We are not interested in creating a complete game (i.e., another SimCity) but rather in developing a plug'n play style framework that supports a variety of game genres and game choices. This is not to downplay the importance of initial and ongoing education for the patient and the family but to extend the impact of games past this initial stage.

Health games in this context then need to satisfy the following criteria: adaptive to the player, change over time, record data, connect to health professionals, provide variety, and provide player choice.

\section{Game Framework for Long Term Use}

Games designed to be used as part of a long term treatment strategy are different from those of games meant for learning about a newly diagnosed disorder. First, one can anticipate that access to the game is a high priority, likely on both mobile and static platforms. Second, individual games must be personalized and adaptable over time. Third, novelty and interest levels must be maintained over the time period, perhaps by rewarding the child with new games a long the way. The game framework we have developed is based on the following design guidelines:

1. A common overall scenario for all players with individualized games accessed through that scenario. A simple communication protocol defines message passing between individual games and the common scenario 


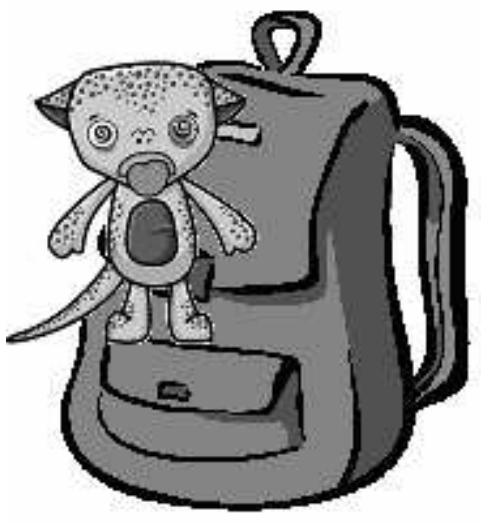

Figure 3a. Pet in need of attention.

2. Plug'n play components so that games can be added and removed from the scenario in a very simple manner.

3. Inclusion of existing non-health related games chosen by the child

4. Adaptation of games for use in health context

5. New games that are designed to reusable and reconfigurable that can be tailored dynamically for different disorders and different treatments

6. Database at the server controlled by the common scenario that allows game instances to be generated dynamically using data from user and disorder profiles and that captures user actions for later analysis.

Overall Scenario: The overall background scenario is the game driver. We have chosen an interactive cityscape of nine city blocks, in Flash, through which the player navigates to choose activities. Figure 2 shows an opening scene in the Halifax version of the cityscape. The player returns to the street level between activities and at the street level the player has access to global health information, reminder boards, a clock, and a backpack of tools. Each game component communicates directly with the cityscape driver and the cityscape communicates directly to the databases at the server to instantiate games, provide personalized information to the games as requested, and to record game interactions in the database. Within this framework we plug in the following games: existing games as-is, modified games, games specifically developed for this framework, and roleplaying text adventure games.

The only game directly connected to the cityscape is the players' personal pet, kept in the player's backpack. If the player neglects to tend to the needs of the pet, such as feeding good food, taking medication,

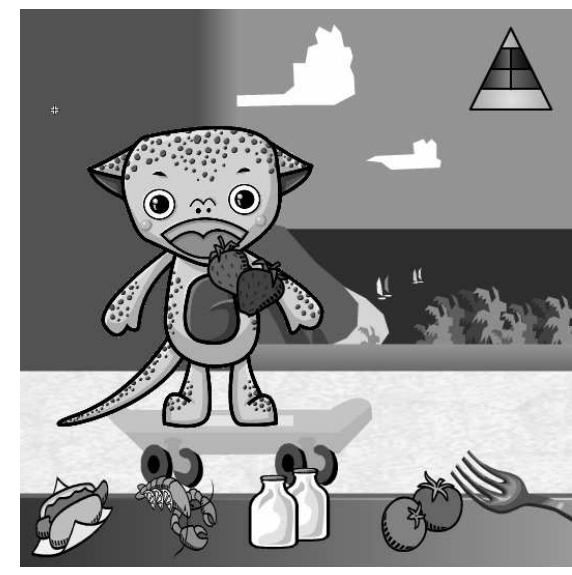

Figure 3b. Feeding the pet.

or practising relaxation techniques at appropriate times during the day the pet begins to look ill, as shown in Figure 3a and can be revived by feeding as in Figure 3b. The food choices that are presented during the play of this game are determined on the fly and may be used by the child to record daily food intake as well as in the play of the game.

Plug'n Play: Within the context of the overall scenario, individual players make choices about activities either by entering a building or opening their backpack. For example, entering the library might result in access to the IBD web site, entering their house could start a text based role playing game, while entering the Children's Hospital might start up one of the user's own games. The actual activities at any site are individualized and driven by the user profile so that the activity that results from entering a given building may be different for different players. All of the component games are designed or wrapped to comply with a simple set of http and xml based protocols. This way, new games can be easily added and games can be easily removed to provide individualized experiences and allow for renewal of the scenario over time and as interests change.

Existing games of the child's preference are included where feasible in the scenario for use as distraction therapy as needed for coping with pain or treatments, reducing anxiety, or as rewards

Adaptation of Other Games: We have been successful in adapting games for health purposes from entertainment games, i.e., making variations of games that are readily available, such as airplane shooters or quest like games. This approach has several advantages; cost savings, higher quality games, more 


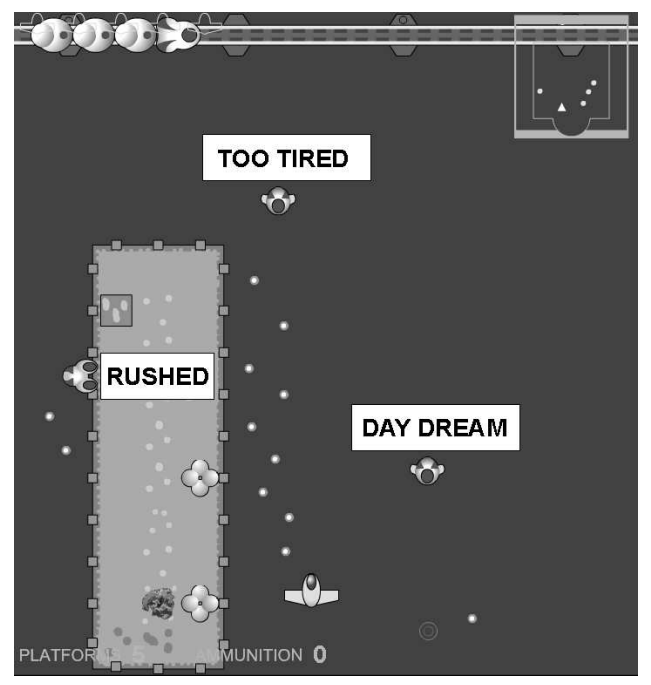

Figure 4a. Airfox played to reinforce relaxation techniques.

complex games, and the ability to individualize or personalize the games. Instances of the adapted game are generated at run time to incorporate specific health content using the database and user's profile. This means that the play of the game remains consistent across its use for different health conditions but that the content can reflect specific treatment conditions, e.g., IBD, asthma, or diabetes, for different users, e.g., by language, by age, or by skill level

By way of example, we have adapted a widely available flash game, AirFox [1], for more general use by inserting a "streamer" object that attaches itself to one class of attack ships plus a function to output the results of events on this object. One of the properties of the inserted object is to display a short text string to individual ships. As the game is played, text from the database is bound dynamically to these objects and the player uses this information to pick which ships to shoot. In Figure 4a we see the user of streamers for relaxation treatments and in Figure $4 \mathrm{~b}$ we see the user of the same streamers for reinforcement of healthy eating. The results of the user actions are stored in the database and the user profile is updated after each episode of play. The manner and sequence of playing of the game is not altered from its original form but the insertion of health specific content can be used to support a treatment agenda, by reinforcing choices, presenting treatment options, providing timely reminders of treatment, or recording of treatment specifics. Furthermore, the game plays the same whether the content is for asthma or diabetes and whether in English, French or Chinese.

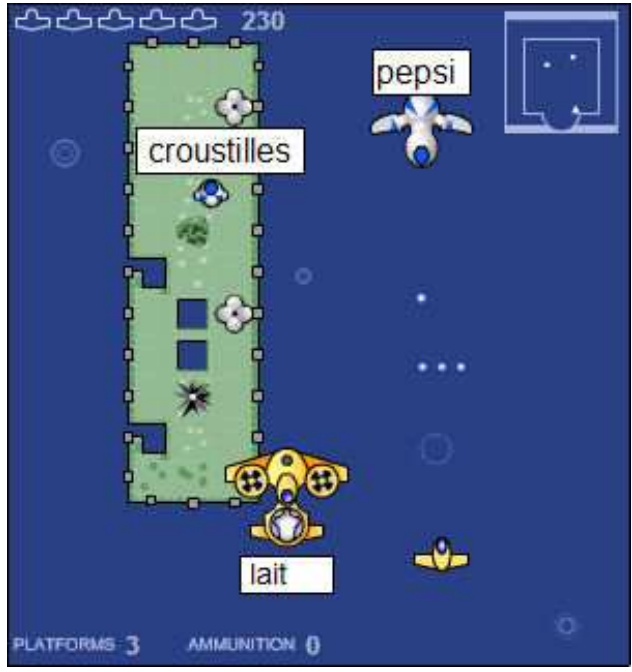

Figure 4b. Airfox played to reinforce health eating.

New Multi-use Games: Although not our primary focus, we continue to develop simple reusable games as prototypes for use in our overall scenario. As an example, a question-answer style Flash game, Leopardy, shown in Figure 5a and Figure 5b, was developed in Flash initially as a Q\&A game with questions and answers drawn from the database to check the understanding of the educational component. It has become more useful as a way of monitoring treatment specifics (selecting energy or pain levels, for example) and reinforcing behaviour by presenting choices (choose a relaxation image or last test result, for example) to the child during the course of treatment. Since the questions and the response choices are stored as Unicode text separately from the game they can be used for a wide variety of purposes and in multilingual versions. For instance, our version of Leopardy plays in English, French, and Chinese.

Text Engine games: We are currently developing a series of interactive text adventure style games for role playing using the Inform Z-machine [18] for natural language style interaction. Using this text game engine we have developed a role playing scenario for parents with children with chronic pain. These parents need to be able to determine within a short time frame whether the symptoms presented to them necessitate keeping their child home from school. The scenario takes place in a simple kitchen with a window to the street and a clock that starts at $8 \mathrm{am}$. The child character comes down the stairs and the parent initiates a dialog which the Inform engine parses and creates appropriate responses. The parent must make a decision before the bus appears in the 


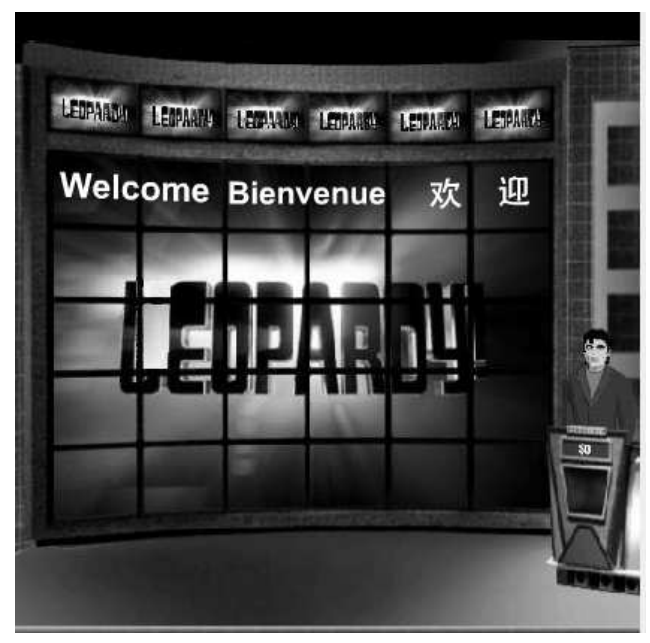

Figure 5a. Leopardy can be played in English, French, and Chinese.

window at $8: 15$. While this first instance is targeted at care givers the second instance will be the scenario from the child's perspective.

Database Layer: The data layer has two main databases, user database and game database. The user database contains user profile and use data while the game database contains rules and content for each game available. All of the games are connected to the data base layer.

The player profile includes treatment parameters, user characteristics, user preferences, game scores, and game interactions and monitoring information. This data is used not only to drive the individual instances of the games but by coaches and clinicians to be apprised of treatment compliance and difficulties experienced by the child during the course of the treatment phase. Coaches will be able to access this data as part of their regular meetings and researchers will have access to the data for analysis in longitudinal studies.

The game database contains parameters about the run time environment of each game, including parameters that are needed from a user profile, disorder specific content, and multilingual information.

\section{Summary}

This architecture described provides support for children and families to engage in games and gamelike scenarios to reinforce lessons and skills and to encourage compliance and monitoring of long term treatments for chronic disorders like diabetes or IBD.

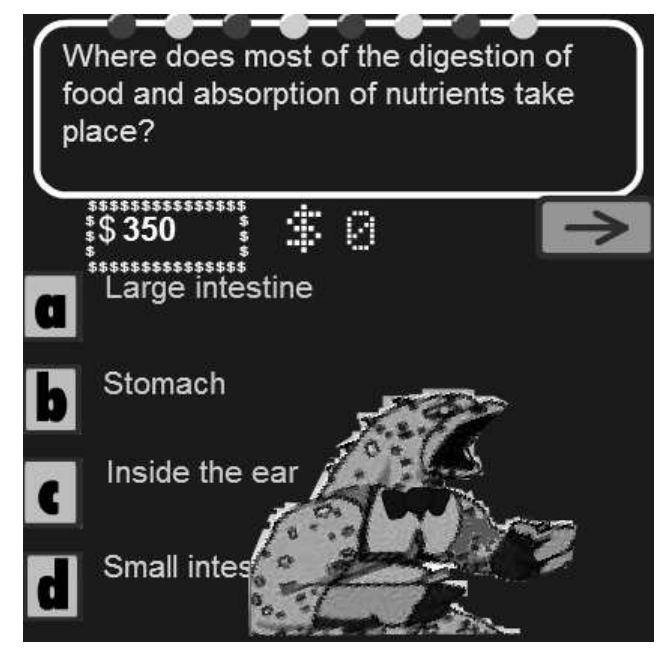

\section{Figure $5 b$. Leopardy is a flexible Q\&A style game.}

This framework has been designed to support and encourage repeated use over long periods by including personalization, replacement of games, continual recording of data, and links to health professionals.

Coaches and health researchers can use this data to connect with children who are not dealing well with their treatment in a timely fashion and later for analysis of outcomes. The underlying hypothesis is that patients will be more engaged in the management of their treatment using the game than they would be using other more traditional recording tools. User profile information is stored for each player, so that the game content and play style can be tailored to the needs of individual players. Coaches and clinicians can make use of the data collected throughout the game, including interactions with the game, choices made, and time and duration of play. This data can be used indirectly to monitor the progress of the patient and directly to intervene as needed for support or additional feedback. Health researchers can use the data for clinical trials evaluating the efficacy of treatments with and without game components.

Producing reusable classes of educational games can have a significant impact on time and cost of game development and may support the building of communities of users. Furthermore, this platform supports continual monitoring as well as follow-up analysis, i.e., both longitudinal studies and clinical trials. The platform has been designed to support both desktop/laptop and mobile PDA access through the Web-based server and choice of development languages Flash, C\#, and TinyGL. Future development work includes building support tools for easier modification of games before beginning trials 
with patients. We are at the beginning of the next phase which is to evaluate both individual games and usefulness to patients and health care providers over extended periods. We are planning these studies in cooperation with the local Children's Hospital.

\section{References}

[1] Airfox. (2005). Available Online at [http://www.thepcmanwebsite.com/media/air_fox/airf ox.shtml].

[2] Bronkie the Bronchiasaurus. (2005). Asthma HealthWorks. Available Online [www.prairiepublic.org/features/healthworks/asthma].

[3] Chapman, M.L. (1999). Situated, Social, Active: Rewriting Genre in the Elementary Classroom. Written Communication, 16(4), 469-490.

[4] Cooper, J. \& Weaver, K.D. (2003). Gender and Computers: Understanding the Digital Divide. Mawah, NJ: Lawrence Erlbaum Associates.

[4] Das, D., Grimmer, K., Sparnon, T., McRae, S. \& Thomas, B. (2005). The Efficacy of Playing a Virtual Reality Game in Modulating Pain for Children with Acute Burn Injuries: A Randomized Controlled Trial. BMC Pediatrics, 4(27).

[5] Dyck J., Pinelle, D., Brown, B. \& Gutwin, C. (2003). Learning from Games: HCI Design Innovations in Entertainment Software. In Proceedings of Graphics Interface, Halifax, Canada, 105-112.

[6] Family Help. (2005). Online at [www.bringinghealthhome.com].

[7] Games for Health. (2005). Available online at [www.gamesforhealth.org].

[8] Glucoboy. (2003). Description available online at: [www.diabetesincontrol.com/issue 173/np.shtml].
[9] Hoffman, H., Doctor, J., Patterson, D., Carrougher, G. \& Furness, T. (2000). Virtual Reality as an Adjunctive Pain Control during Burn Wound Care in Adolescent Patients. Pain, 85(1-2), 305-309.

[10] Holden, G., Bearison, D., Rode, D., Rosenberg, G. \& Fishman, M. (1999). Evaluating the Effects of a Virtual Environment (STARBRIGHT World) with Hospitalized Children. Research on Social Work Practice, 9(3), 365-382.

[11] Palermo, T., Valenzuela, M. \& Stork, P. (2004). A Randomized Trial of Electronic versus Paper Pain Diaries in Children: Impact on Compliance, Accuracy, and Acceptability. Pain, 107(3), 213-219.

[12] Patel, A. (2005). Distraction with Handheld Video Game is an Effective Anxiolytic. Reported in Anesthiology News. March 2005. Available Online at [www.anethesiologynews.com]

[13] SAGE. (2003). Simulation and Advanced Gaming Environments for Learning. Available Online at [www.sageforlearning.ca]

[14] STARBRIGHT World. (2005). Available Online at [www.starbright.org]

[15] Turkle, S. (1995). Life on the screen: Identity in the age of the Internet. New York, NY: Simon \& Schuster.

[16] Watters, C. \& Duffy, J. (2004). Metalevel Analysis of Motivational Factors for Interface Design. In K. Fisher, Erdelez, S. and McKechnie, E.F. (Ed.), Theories of Information Behavior: A Researcher's Guide. Medford, NJ: ASIST (Information Today, Inc.).

[17] Wolf, M.J.P. (2001). The Medium of the Video Game. Austin, TX: University of Austin Press.

[18] Z-machine. (2005). Inform. Available online at [www.inform-fiction.org]. 